\title{
Multiobjective economic MPC of constrained non-linear systems
}

ISSN 1751-8644

Received on 24th September 2015

Revised on 19th February 2016

Accepted on 24th March 2016

doi: 10.1049/iet-cta.2015.0947

www.ietdl.org

\author{
Defeng He $\mathrm{H}^{1,2 凶}$, Jing Sun ${ }^{2}$, Wei Chen ${ }^{3}$ \\ ${ }^{1}$ College of Information Engineering, Zhejiang University ofTechnology, Hangzhou 310023, People's Republic of China \\ ${ }^{2}$ Department of Naval Architecture and Marine Engineering, University of Michigan, Ann Arbor, MI 48109, USA \\ ${ }^{3}$ School of Electrical Engineering and Automation, Hefei University of Technology, Hefei 230009, People's Republic of China \\ 凶-mail: hdfzj@zjut.edu.cn
}

\begin{abstract}
This study proposes an alternative utopia-tracking multiobjective economic model prediction control scheme of constrained non-linear systems with guaranteed asymptotic stability and convergence of average performance. The proposed scheme minimises the distance of its cost function vector to a vector of independently minimised objectives evaluated at ultimate transient operation, i.e. ultimate utopia point. Recursive feasibility and stability of the scheme are established by a closed-loop optimisation dual-mode formulation. Moreover, convergence of the closed-loop average performance is established in the context of multiobjective optimisation. The theoretical results are illustrated by two examples of chemical processes.
\end{abstract}

\section{Introduction}

Economic model predictive control (EMPC) has recently received much attention due to its ability in integrating real-time process economic optimisation and feedback control into an optimal control framework $[1,2]$. In contrast to the traditional MPC where quadratic cost functions are often used, EMPC directly utilises general economic criteria as stage costs. Using different assumptions and/or additional constraints, several EMPC schemes were proposed to address the critical challenges of designing controllers with guaranteed stability and optimal economic performance [3-13]. In particular, Rawlings et al. [14] summarised recent development on the stabilising design of EMPC and [1] reviewed the recent results on closed-loop system stability and performance issues on the various EMPC schemes in the literature.

In many EMPC applications, the economic performance encapsulates many control objectives, such as tracking, profitability, sustainability, etc. The cost criteria representing these objectives are generally incompatible and in conflict such that no unique (globally) optimal solution to the multiobjective optimisation problem (MOOP) is attainable $[15,16]$. In order to solve this type of MOOPs, an accepted solution is to find a set of non-inferior or non-dominated Pareto optimal solutions that optimise the multiple cost criteria as much as possible [16]. In this sense, multiobjective EMPC (MO-EMPC) with multiple economic criteria often yields a number of different Pareto optimal EMPC controllers that reflect different tradeoffs among the competing economic criteria and provide implementers with different design candidates that can accommodate different control requirements. However, it is not easy to determine which Pareto optimal EMPC controllers are preferred over others, unless some additional procedures are followed.

A well-known procedure for dealing with multiple competing objectives is to use the weighted sum approach [16], where a single objective optimisation problem is formulated by combining the multiple objectives through a weighted sum so that it can be conveniently solved by conventional single objective optimisation methods. In general, the weights need to be tuned and even to be adjusted at each sampling time [17-19] for desired responses. However, tuning the weights to select a preferred Pareto solution is conceptually easy but in practice suffers from some intrinsic drawbacks [19]. For instance, a systematic variation of weights does not necessarily result in a uniform distribution of Pareto solutions.
To overcome the difficulties in weight selection, Flores-Tlacuahuac et al. [20] proposed a steady-state utopia-tracking MO-MPC scheme ensuring asymptotic stability by a terminal constraint and a strong duality assumption [21]. By minimising the distance of a set of stage cost functions to its steady-state utopia point, the proposed controller does not need to compute online the Pareto optimal solution set, instead it minimises multiple objectives automatically without requiring weights to be adjusted or expert knowledge to be used. To improve this MO-MPC in terms of computational load and feasible state set, in [22] we used control Lyapunov functions [23] to propose a dual-mode utopia-tracking MO-MPC scheme for input-affine non-linear systems, where a terminal region constraint, a varying prediction horizon and an explicit closedloop optimisation were exploited to ensure recursive feasibility and stability.

One of the main features of the utopia-tracking MO-EMPC is that the resulting closed-loop system may exhibit a non-converging or even cyclic behaviour [24]. Hence, Maree and Imsland [15] introduced the concept of dynamic utopia point for periodic processes and presented a dynamic utopia-tracking MO-EMPC design for cyclic operation, where the controller minimises the average cyclic performance defined by a cost distance from the dynamic utopia point. Recursive feasibility of the scheme was achieved using a cyclic terminal constraint; however, conditions for closed-loop stability were not established.

In EMPC, the economic cost $L(x, u)$ may not be positive definite with respect to any steady-state point $\left(x_{s}, u_{s}\right)$. Consequently, it may occur that $L(x, u)<L\left(x_{s}, u_{s}\right)$ for some transient points $(x, u)$ [7]. This typical phenomenon of EMPC can also be expected in MO-EMPC that optimises multiple economic criteria. Hence, it is possible that the utopia points defined by average performance of ultimate transient processes outperform those defined by steadystate ones. Although the non-converging behaviour resulted from the utopia-tracking strategy can be accepted in some applications [15], convergence to an equilibrium is essential in many other cases where improved economics should not come at the cost of losing convergence. Hence, one of the important problems of utopia-tracking MO-EMPC is to find conditions under which the closed-loop system converges to an equilibrium point. Moreover, to our best knowledge, the existing utopia-tracking MO-EMPC schemes have not addressed the average performance topic in the context of economic MOOP. 
Here we proposed an alternative utopia-tracking MO-EMPC for constrained continuous-time non-linear systems. An alternative utopia point is defined to cope with economic multiobjective optimisation. Then a utopia-tracking closed-loop optimisation problem is formulated by choosing the cost function as the distance of the cost functions to the new utopia point. Recursive feasibility and stability of the MO-EMPC are established under the assumptions of controllability, a terminal region and a family of local controllers defined on the terminal region. Moreover, the average performance of each cost function is shown to converge to the compromised point. Compared with the steady-state utopia-tracking strategies, e.g. [21], the first contribution of this work is to propose an alternative definition of utopia point for economic optimisation, i.e. the ultimate utopia point, which may improve the average performance of economic criteria. The second contribution of this work is that recursive feasibility and stability of the proposed MO-EMPC are established in the dual-mode framework using the controllability and terminal region conditions, which increases the size of the set of initial feasible states. These features will be illustrated by an example of chemical process control.

Notation: Let $\mathbf{I}_{>0}$ denote the set of non-negative integer numbers, $\mathbf{I}_{\geq a}$ be the set $\left\{i \in \mathbf{I}_{\geq 0}: i \geq a\right\}$ and $\mathbf{I}_{a: b}$ be the set $\left\{i \in \mathbf{I}_{\geq 0}: a \leq i \leq b\right\}$ for some $a \in \mathbf{I}_{>0}$ and $b \in \mathbf{I}_{>0}$. Denote by $A \backslash B=\{x: x \in A, x \notin B\}$ the difference of two given sets $A \subseteq R^{n}$ and $B \subseteq R^{n}$. Notation $\|\cdot\|$ is a standard two-norm and $\mathbf{u}_{k: k+N}$ is a (piecewise) continuous signal $u(t)$ for $t \in\left[t_{k}, t_{k+N}\right]$. The solution to a system at time $t$ from the initial state $x_{0}$ for an input $\mathbf{u}_{0: N}$ is denoted as $x(t)=\varphi\left(t ; x_{0}, \mathbf{u}_{0: N}\right)$.

\section{Problem description and preliminaries}

Consider continuous-time non-linear systems of the form

$$
\dot{x}(t)=f(x(t), u(t)), \quad x(0)=x_{0}
$$

where the state $x \in X \subseteq R^{n}$, control $u \in U \subset R^{m}$ and function $f$ : $X \times U \rightarrow X$ is locally Lipschitz continuous in $(x, u) . x_{0}$ is the initial state. Let $O=\left\{x_{s} \in R^{n} \mid \exists u_{s} \in U\right.$ s.t. $\left.0=f\left(x_{s}, u_{s}\right)\right\}$ be the admissible equilibrium set of the system, which is assumed to be non-empty. The constraints $X$ and $U$ are compact sets satisfying $O \subset X$. We assume that the states can be measured at each time instance of the discrete time sequence $\left\{t_{k}, k \in \mathbf{I}_{>0}\right\}$ with $t_{k}=t_{0}+$ $k \delta$ and a sampling period $\delta>0$. For simplicity, let $t_{0}=0$. Given a finite $N \in \mathbf{I}_{>1}$ and a compact set $X_{T} \subseteq X$ with $O \subset X_{T}$, an input $\mathbf{u}_{0: N}$ is said to be feasible for $x_{0} \subseteq X$ if $u(t) \in U, \varphi\left(t ; x_{0}, \mathbf{u}_{0: N}\right) \in X$ and $\varphi\left(t_{N} ; x_{0}, \mathbf{u}_{0: N}\right) \in X_{T}$ for all $t \in\left[0, t_{N}\right]$, where $\varphi$ satisfies (1).

Consider a set of economic criteria with $l \in \mathbf{I}_{\geq 2}$ general stage costs of the form $L_{j}: X \times U \rightarrow R, j \in \mathbf{I}_{1: l}$. We are interested in the multiobjective optimal control problem (OCP) defined over a prediction horizon of $\mathrm{N}$-time periods,

$$
\begin{aligned}
& \min _{\mathbf{u}_{k: k+N}}\left\{J_{1}\left(x_{k}, \mathbf{u}_{k: k+N}\right), \ldots, J_{l}\left(x_{k}, \mathbf{u}_{k: k+N}\right)\right\} \\
& \text { s.t. } \quad \dot{x}(t)=f(x(t), u(t)), \quad \forall t \in\left[t_{k}, t_{k+N}\right] \\
& x(t) \in X, \quad u(t) \in U, \quad \forall t \in\left[t_{k}, t_{k+N}\right] \\
& x\left(t_{k}\right)=x_{k}, \quad x\left(t_{k+N}\right) \in X_{T}
\end{aligned}
$$

where the cost function $J_{j}(x, \mathbf{u})$ is defined as

$$
J_{j}\left(x_{k}, \mathbf{u}_{k: k+N}\right)=\int_{t_{k}}^{t_{k+N}} L_{j}(x(t), u(t)) \mathrm{d} t
$$

for all $j \in \mathbf{I}_{1: l}$, initial condition $x_{k}$ is the state at sampling time $t_{k}$ and $X_{T}$ is a terminal set. Let $L(x, u)=\left[L_{1}(x, u), \ldots, L_{l}(x, u)\right]^{T}$ be a stage cost vector and $J(x, \mathbf{u})=\left[J_{1}(x, \mathbf{u}), \ldots, J_{l}(x, \mathbf{u})\right]^{T}$ be a cost function vector. Since the economic criteria $L_{j}(x, u)$ are generally conflicting, the optimality of the multiobjective OCP (2) is typically defined in the sense of Pareto optimality based on cost vector dominance $[15,16]$.
Definition 1 [15]: At time $t_{k}, k \in \mathbf{I}_{\geq 0}$, a feasible solution $\hat{\mathbf{u}}_{k: k+N}$ to (2) is Pareto optimal if there is no other feasible solution $\mathbf{u}_{k: k+N}$ such that $J_{j}\left(x_{k}, \mathbf{u}_{k: k+N}\right) \leq J_{j}\left(x_{k}, \hat{\mathbf{u}}_{k: k+N}\right)$ for all $j \in \mathbf{I}_{1: l}$ and $J_{i}\left(x_{k}, \mathbf{u}_{k: k+N}\right)<J_{i}\left(x_{k}, \hat{\mathbf{u}}_{k: k+N}\right)$ for at least one $i \in \mathbf{I}_{1: l}$.

Unlike static multiobjective optimisation where the Pareto optimal solutions depend solely on the cost functions, Pareto optimality of (2) is dependent on the initial condition $x_{k}$ at each time $t_{k}$ because of its receding horizon feature. In general, constructing a Pareto optimal set is computationally exhaustive, making it impractical for real-time control. To overcome the difficulties in computing solutions for the MO-EMPC problem given by (2) and (3), feasible methods have been proposed to directly use the stage costs $L_{j}(x, u)$ to design MO-EMPC, such as the steady-state utopia-tracking strategy [21].

Definition 2 [21]: A vector $L_{s}^{*} \in R^{l}$ is the steady-state utopia point of system (1) with respect to the cost vector $L(x, u)$ if and only if for each $j \in \mathbf{I}_{1: l}$, we have

$$
L_{s, j}^{*}=\min _{(x, u) \in X \times U}\left\{L_{j}(x, u) \quad \text { s.t. } \quad f(x, u)=0\right\}
$$

with the optimal solution $\left(x_{s, j}^{*}, u_{s, j}^{*}\right)$.

Definition 3 [21]: A state $x_{s}^{c} \in O$ is a compromised steady state of system (1) with respect to $L(x, u)$ if and only if it satisfies

$$
\left(x_{s}^{c}, u_{s}^{c}\right)=\arg \min _{(x, u) \in X \times U}\left\{\left\|L(x, u)-L_{s}^{*}\right\| \quad \text { s.t. } \quad f(x, u)=0\right\}
$$

with the compromised point $L\left(x_{s}^{c}, u_{s}^{c}\right)$, where $L_{s}^{*}=\left[L_{s, 1}^{*}, L_{s, 2}^{*}, \ldots\right.$, $\left.L_{s, l}^{*}\right]^{\mathrm{T}}$.

The idea of the utopia-tracking strategy is to minimise a distance of its cost vector to the steady-state utopia point $L_{s}^{*}$. The main advantage of this strategy is that it can make a trade-off between multiple objectives of interest automatically with no need to construct the entire Pareto front or select weights. In general, each stage cost $L_{j}(x, u)$ is transformed to be dimensionless in order to improve computational performance, especially when each element of $L(x, u)$ has different unit.

Remark 1: If each $L_{j}(x, u)$ is positive definite with respect to its optimal equilibrium point $\left(x_{s, j}^{*}, u_{s, j}^{*}\right)$, i.e. $L_{j}(x, u) \geq 0$ for $(x, u) \in X \times U$ and $L_{j}(x, u)=L_{s, j}^{*}=0$ if and only if $(x, u)=$ $\left(x_{s, j}^{*}, u_{s, j}^{*}\right)$, then the steady-state utopia point $L_{s}^{*}$ is unachievable for the conflicting cost functions $L_{j}(x, u)$ with $j \in \mathbf{I}_{1: l}$. However, this property will not hold for general economic criteria $L(x, u)$ since there usually exist some points $(x, u) \in X \times U$ such that $L_{j}(x, u) \leq L_{s, j}^{*}$ for $j \in \mathbf{I}_{1: l}$. Recall that for single objective EMPC, the average performance of $L_{j}(x, u)$ is achieved as

$$
\lim _{k \rightarrow \infty} \sup \frac{1}{t_{k}} \int_{0}^{t_{k}} L_{j}\left(\phi\left(t ; x(0), \mathbf{u}_{0: t_{k}}\right), u(t)\right) \mathrm{d} t \leq L_{j}\left(x_{s, j}^{*}, u_{s, j}^{*}\right)=L_{s, j}^{*}
$$

under some feasibility conditions $[1,7]$. The aforementioned facts suggest that the utopia point defined by (4) may not be the best choice of utopia point in the context of economic criteria optimisation.

\section{Utopia points for economic criteria optimisation}

In the context of economic optimisation, the average performance of each $L_{j}(x, u)$, measured over an enough long time horizon, may be less than its economically optimal steady-state performance and is dependent on the initial state $x_{0}$. Similarly, it is known from 
Definition 1 that at each sampling time $t_{k}, k \in \mathbf{I}_{\geq 0}$, the Pareto optimal front of the OCP (2) also depends on the boundary condition $x\left(t_{k}\right)=x_{k}$. Moreover, the control optimising the economic criteria does not necessarily lead to a trajectory which converges to some steady-state points [11]. Therefore the closed-loop system may exhibit behaviour such as a periodic orbit [24]. For many applications, a desirable property of an optimal control is that the closed-loop system will remain at some economically optimal steady-state points, such as the compromised point $\left(x_{s}^{c}, u_{s}^{c}\right)$, after an appropriate transient operation. Clearly, the control input $u(t) \equiv u_{s}^{c}$ has this desirable property if the initial state is picked as $x_{s}^{c}$. Otherwise, for any $x_{0} \in X$, a general condition ensuring this desirable property is to use a terminal constraint

$$
\phi\left(t_{T} ; x_{0}, \mathbf{u}_{0: T}\right)=x_{S}^{\text {opt }}
$$

where $x_{s}^{\text {opt }} \in O$ is an economically optimal steady state with respect to some economic criteria of interest and $t_{T}=T \delta, T \in \mathbf{I}_{\geq 1}$, is an ultimately transient time after which the closed-loop system enters the steady state after starting from $x_{0}$. In this sense, condition (7) generalises the case where the boundary condition (2) is directly set as the steady state. Note that for cyclic processes, (7) can be substituted by the cyclic terminal constraint in [15]. Motivated by this dynamic utopia point for cyclic operation, a new utopia point for non-cyclic operation is defined, which attempts at finding an optimal period length such that average performance measured over this period may be less than the steady-state optimal performance for any $x_{0} \in X$.

Definition 4: A vector $L_{u}^{*} \in R^{l}$ is an ultimate utopia point of system (1) with respect to $L(x, u)$ if and only if

$$
\begin{gathered}
L_{u, j}^{*}=\min _{x_{0}, t_{T}, u_{0: T}} J_{j}\left(x_{0}, \mathbf{u}_{0: T}\right) / t_{T} \\
\text { s.t. } \quad \dot{x}(t)=f(x(t), u(t)), \quad x\left(t_{0}\right)=x_{0} \\
x(t) \in X, u(t) \in U, \quad x_{0} \in X, \quad \forall t \in\left[t_{0}, t_{T}\right] \\
x\left(t_{T}\right)=x_{s, j}^{*}, \quad t_{T} \geq 0
\end{gathered}
$$

for each $j \in \mathbf{I}_{1: l}$, where $x_{s, j}^{*} \in O$ is the optimal steady state with respect to $L_{j}(x, u)$.

Remark 2: In Definition 2, the initial state $x_{0}$ and time window $t_{T}$ are chosen as the decision variables of (8) in addition to $\mathbf{u}_{0: T}$. The optimal initial state and period length enable us to evaluate an optimal average performance of each $L_{j}(x, u)$ with respect to $X$ and time range. For computational considerations, however, $t_{T}$ can be limited to some upper bound. For instance, we can use a priori information of the bound of $t_{T}$ satisfying (7) to solve (8). It is noted that the solution of (8) is obtained offline and it does not increase the computational demand of solving MPC actions online.

Proposition 1: Consider the optimisation problems (4) and (8). If $t_{T}^{*}>0$, we have $L_{u, j}^{*} \leq L_{s, j}^{*}$ for each $j \in \mathbf{I}_{1: l}$. Else, we have $L_{u, j}^{*}=$ $L_{S, j}^{*}$ if $t_{T}^{*} \rightarrow 0$.

Proof: Consider $L_{j}(x, u)$ for any $j \in \mathbf{I}_{1: l}$. Let $\left(x_{s, j}^{*}, u_{s, j}^{*}\right)$ with $L_{s, j}^{*}$ and $\left(x_{0, j}^{*}, t_{T}^{*}, \mathbf{u}_{0: T}^{*}\right)$ with $L_{u, j}^{*}$ be the solutions to (4) and (8) for $j \in \mathbf{I}_{1: l}$, respectively. Using $x_{s, j}^{*}, u_{s, j}^{*}$ and $t_{T}^{*}$, we construct a solution candidate $\left(x_{s, j}^{*}, t_{T}^{*}, \tilde{\mathbf{u}}_{0: T}\right)$ to $(8)$ with $\tilde{\mathbf{u}}_{0: T}=u(t) \equiv u_{s, j}^{*}$ for $t \in\left[0, t_{T}^{*}\right]$. It is clear that this candidate satisfies the constraints (8b)-(8d) with $x_{0}=x_{s, j}^{*}$. Since the pair $\left(x_{s, j}^{*}, u_{s, j}^{*}\right)$ is a steady-state point, the candidate $\left(x_{s, j}^{*}, t_{T}^{*}, \tilde{\mathbf{u}}_{0: T}\right)$ is a feasible solution to (8). Substituting it to (4), we have $J_{j}\left(x_{s, j}^{*}, \tilde{\mathbf{u}}_{0: T}\right)=t_{T}^{*} L_{s, j}^{*}$. From the definition of $L_{u, j}^{*}$, we derive that $L_{u, j}^{*} \leq J_{j}\left(x_{s, j}^{*}, \tilde{\mathbf{u}}_{0: T}\right) / t_{T}^{* *}=L_{s, j}^{*}$.
Consider the case of $t_{T}^{*} \rightarrow 0$. Since $L_{j}(x, u)$ is continuous on its arguments, we have

$$
\lim _{t_{T} \rightarrow 0} J_{j}\left(x_{0}, \mathbf{u}_{0: T}\right)=\lim _{t_{T} \rightarrow 0} \int_{0}^{t_{T}} L_{j}(x(t), u(t)) \mathrm{d} t=L_{j}\left(x_{0}, u_{0}\right) .
$$

Then from (4) and (8), it is concluded that the optimal values $L_{u, j}^{*}$ and $L_{s, j}^{*}$ are identical when $t_{T}^{*} \rightarrow 0$.

Consider the steady-state utopia-tracking OCP

$$
\mathbf{u}_{s}^{*}=\arg \min _{u}\left\{J_{s}(x, \mathbf{u}) \text { s.t. }(2 b)-(2 d)\right\}
$$

where $x$ is the state at time $t_{k}$ and $J_{s}(x, \mathbf{u})$ is the steady-state utopia-tracking objective function

$$
J_{S}(x, \mathbf{u})=\int_{0}^{t_{N}}\left\|L(x(t), u(t))-L_{s}^{*}\right\| \mathrm{d} t .
$$

Define the ultimate utopia-tracking objective function

$$
J_{u}(x, \mathbf{u})=\left\|\int_{0}^{t_{N}}\left[L(x(t), u(t))-L_{u}^{*}\right] \mathrm{d} t\right\|
$$

and the corresponding ultimate utopia-tracking OCP is formulated as

$$
\mathbf{u}_{u}^{*}=\arg \min _{u}\left\{J_{u}(x, \mathbf{u}) \text { s.t. }(2 b)-(2 d)\right\} .
$$

Define two time-average performances as

$$
J_{u}^{a}=\frac{1}{t_{N}} \int_{0}^{t_{N}} L\left(x(t), u_{u}^{*}(t)\right) \mathrm{d} t, \quad J_{s}^{a}=\frac{1}{t_{N}} \int_{0}^{t_{N}} L\left(x(t), u_{s}^{*}(t)\right) \mathrm{d} t .
$$

We have the following result on the steady-state utopia point and the ultimate utopia point:

Proposition 2: Suppose that the optimisation problems (9) and (12) are feasible for the state $x \in X$ and the finite horizon $t_{N}>0$. Then we have

$$
\begin{aligned}
\left\|J_{u}^{a}-L_{u}^{*}\right\| & \leq\left\|J_{s}^{a}-L_{u}^{*}\right\|, \\
\left\|J_{s}^{a}-L_{s}^{*}\right\| & \leq\left\|J_{u}^{a}-L_{s}^{*}\right\|
\end{aligned}
$$

Moreover, the performance $J\left(x, \mathbf{u}_{u}^{*}\right)$ (i.e. $\left.t_{N} J_{u}^{a}\right)$ is no worse than $J\left(x, \mathbf{u}_{s}^{*}\right)$ (i.e. $\left.t_{N} J_{s}^{a}\right)$ if $J_{s}^{a} \geq L_{u}^{*}$.

Proof: Let $\mathbf{u}_{s}^{*}$ be the optimal solution to the problem (9). Then $\mathbf{u}_{s}^{*}$ satisfies (2b)-(2d), which implies that $\mathbf{u}_{s}^{*}$ is a feasible solution to (12). Hence, we have $J_{u}\left(x, \mathbf{u}_{u}^{*}\right) \leq J_{u}\left(x, \mathbf{u}_{s}^{*}\right)$, i.e.

$$
\left\|\int_{0}^{t_{N}}\left[L\left(x(t), u_{u}^{*}(t)\right)-L_{u}^{*}\right] \mathrm{d} t\right\| \leq\left\|\int_{0}^{t_{N}}\left[L\left(x(t), u_{s}^{*}(t)\right)-L_{u}^{*}\right] \mathrm{d} t\right\|
$$

which is equal to (13). Similarly, one can obtain (14).

By contradiction, we assume that $J\left(x, \mathbf{u}_{u}^{*}\right)$ is worse than $J\left(x, \mathbf{u}_{s}^{*}\right)$, i.e.

$$
\int_{t_{0}}^{t_{N}} L_{j}\left(x(t), u_{u}^{*}(t)\right) \mathrm{d} t>\int_{t_{0}}^{t_{N}} L_{j}\left(x(t), u_{s}^{*}(t)\right) \mathrm{d} t
$$

for each $j \in \mathbf{I}_{1: l}$. Being divided by $t_{N}$ and subtracting $L_{u}^{*}$ from both sides of (16), we have

$$
J_{u}^{a}-L_{u}^{*}>J_{s}^{a}-L_{u}^{*} .
$$

Since $J_{s}^{a} \geq L_{u}^{*}$, (17) leads to $\left\|J_{u}^{a}-L_{u}^{*}\right\|>\left\|J_{s}^{a}-L_{u}^{*}\right\|$, which contradicts (13). Therefore, $J\left(x, \mathbf{u}_{u}^{*}\right)$ is no worse than $J\left(x, \mathbf{u}_{s}^{*}\right)$ if $J_{s}^{a} \geq L_{u}^{*}$. 
Remark 3: Fig. 1 illustrates the basic ideas of Propositions 1 and 2, where the larger circle has $L_{u}^{*}$ as the centre and $\left\|J_{s}^{a}-L_{u}^{*}\right\|$ as the radius, while the smaller circle has $L_{s}^{*}$ as the centre and $\left\|J_{s}^{a}-L_{S}^{*}\right\|$ as the radius. Note that $J_{s}^{a}$ is a tangent point of both circles. For simplicity, in this figure we use only two conflicting criteria $L_{1}$ and $L_{2}$ to compute the control actions which meet both criteria in the manner of the utopia solution. From this figure, one can see that $J_{u}^{a}$ is closer to $L_{u}^{*}$ than $J_{s}^{a}$, and it is always in the larger circle but not in the smaller circle. Hence, it is not obtained that $J\left(x, \mathbf{u}_{u}^{*}\right)=t_{N} J_{u}^{a}>t_{N} J_{s}^{a}=J\left(x, \mathbf{u}_{s}^{*}\right)$. In other words, $J\left(x, \mathbf{u}_{u}^{*}\right)$ is no worse than $J\left(x, \mathbf{u}_{s}^{*}\right)$.

\section{$4 \quad$ Multiobjective EMPC}

\subsection{Utopia-tracking MO-EMPC algorithm}

Consider the compromised solution $\left(x_{s}^{c}, u_{s}^{c}\right)$ obtained by solving (5) and let $\left(x_{s}^{c}, u_{s}^{c}\right)=(0,0)$ for simplicity.

Assumption 1: There exists a locally Lipschitz control law $u=h(x, \theta)$ with $h(0, \theta)=0$ and some parameter $\theta \in R^{q}$ such that the origin of the system (1) in the closed-loop with $u=h(x, \theta)$ is locally asymptotically stable. Moreover, there exists a continuously differentiable Lyapunov function $V: R^{n} \rightarrow R_{\geq 0}$ such that

$$
\begin{array}{r}
\alpha_{1}(\|x\|) \leq V(x) \leq \alpha_{2}(\|x\|) \\
\frac{\partial V(x)}{\partial x} f(x, h(x, \theta)) \leq-\alpha_{3}(\|x\|)
\end{array}
$$

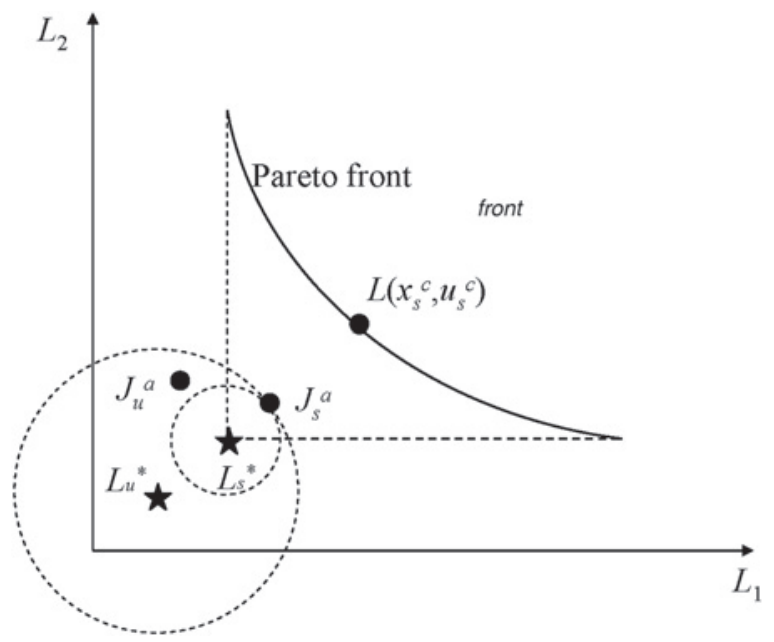

Fig. 1 Basic ideas of Propositions 1 and 2 for two conflicting criteria. Pareto front featuring the conflicting criteria and the compromised point $L\left(x_{s}^{c}, u_{s}^{c}\right)$ are shown for some $\theta$ and all $x \in D$, where $D$ is an open neighbourhood of the origin and $\alpha_{i}, i \in \mathbf{I}_{1: 3}$ are class- $\mathcal{K}$ functions.

Let $\Theta \subseteq R^{q}$ be the parameter set such that Assumption 1 holds for any $\theta \in \Theta$. In the literature, several approaches have been used to select $h(x, \theta)$ and $\Theta$ to satisfy Assumption 1; e.g. feedback linearisation [23], control Lyapunov function [25, 26] and backstepping control [23, 27].

Let $x_{k}$ be the state at sampling time $t_{k}, k \in \mathbf{I}_{>0}$. The ultimate utopia-tracking MO-EMPC problem is formulated as the following dual-mode OCPs

$$
\begin{gathered}
\min _{\mathbf{u}_{k: k+N}} J_{u}\left(x_{k}, \mathbf{u}_{k: k+N}\right) \\
\text { s.t. }\left\{\begin{array}{l}
\dot{x}(t)=f(x(t), u(t)), \quad x\left(t_{k}\right)=x_{k} \\
(x(t), u(t)) \in X \times U, \quad \forall t \in\left[t_{k}, t_{k+N}\right] \\
x\left(t_{k+N}\right) \in X_{T}
\end{array}\right.
\end{gathered}
$$

where the terminal region $X_{T} \subseteq X$ contains the origin in its interior, and

$$
\begin{gathered}
\min _{\theta\left(t_{k}\right) \in \Theta} J_{u}\left(x_{k}, \mathbf{u}_{k: k+N}\right) \\
\text { s.t. } \quad\left\{\begin{array}{l}
\dot{x}(t)=f(x(t), u(t)), \quad x\left(t_{k}\right)=x_{k} \\
u(t)=h\left(x(t), \theta\left(t_{k}\right)\right), \\
(x(t), u(t)) \in X \times U, \quad \forall t \in\left[t_{k}, t_{k+N}\right]
\end{array}\right.
\end{gathered}
$$

where $J_{u}(x, \mathbf{u})$ is given by (11). Then the steps of implementing this strategy are summarised as Algorithm 1.

Remark 4: In Algorithm 1 (Fig. 2), the control input within the first $N$ time samples does not employ the receding horizon nature of MPC. Nevertheless, the optimal control input in Steps 3-5 can be computed by solving (19) online in the fashion of shrinking prediction horizon and then applied to the system (1) in the manner of receding horizon control. Moreover, (20) is a closed-loop optimisation problem due to the state feedback nature of $h(x, \theta)$ and its optimal solution $\theta^{*}\left(t_{k}\right)$ relies on the current state $x\left(t_{k}\right)$, i.e. $\theta^{*}\left(t_{k}\right)=\theta^{*}\left(x\left(t_{k}\right)\right)$. Hence, the controller minimising (20) is a time-invariant state feedback control law.

Algorithm 1 (Fig. 2) implicitly defines an ultimate utopiatracking MO-EMPC controller

$$
u^{m o}(x(t))=\mathbf{u}_{k: k+1}^{*}, \quad \forall t \in\left[t_{k}, t_{k+1}\right], \quad \forall k \in I_{\geq 0}
$$

and the closed-loop system

$$
\dot{x}(t)=f\left(x(t), u^{m o}(x(t))\right), \quad x(0)=x_{0} .
$$

In what follows, we analyse the stability of (22) and the closed-loop average performance of $L(x, u)$.

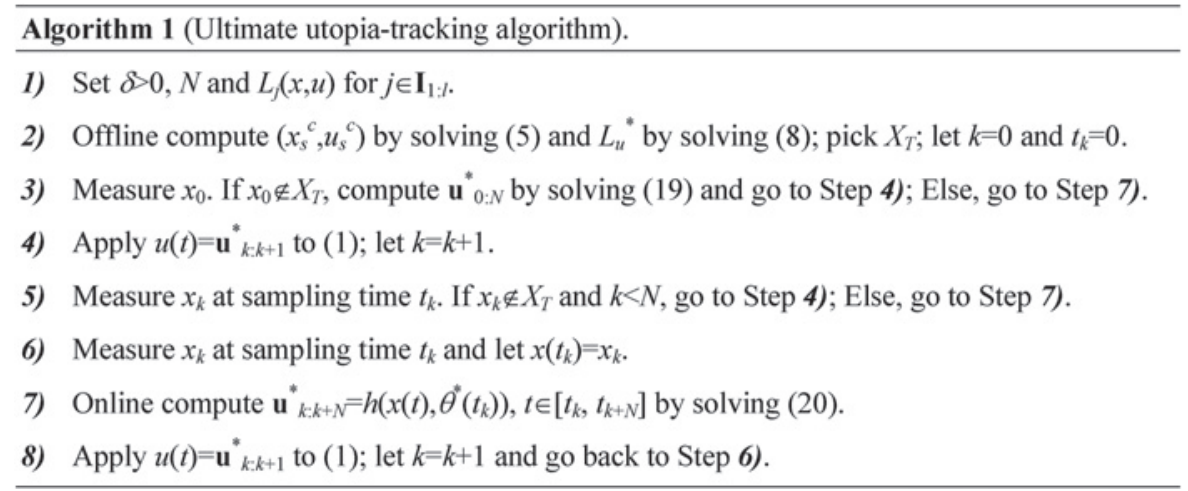

Fig. 2 Ultimate utopia-tracking algorithm 


\subsection{Stability and performance}

In general, the region $D$ is not invariant for the system (1) in the closed-loop with $h(x, \theta)$. We define a level set of $V(x)$ as $\Omega(r)=$ $\left\{x \in R^{n} \mid V(x) \leq r, r>0\right\}$ and it is a compact invariant subset of $D$, containing the origin as its interior. Clearly, the radius $r$ depends on $\theta$. We give a procedure to derive $\Omega(r)$ as large as possible provided that $V(x)$ is known.

Consider the system (1) and define set $X_{h}=\left\{x \in R^{n} \mid \exists \theta \in \Theta\right.$ s.t. $h(x, \theta) \in U\}$. Iteratively increase $r$ from 0 until $\Omega(r)$ is not contained in $X \cap X_{h}$, which yields a preferably largest radius $r_{\max }$. Let $X_{T}=\left\{x \in R^{n} \mid V(x) \leq r_{\max }\right\} \subseteq X$. Then $X_{T}$ is a maximal estimate of invariant sets $\Omega(r)$ of the system (1) in the closed-loop with $h(x, \theta)$ for some $\theta \in \Theta$. Therefore, there exists a $\theta \in \Theta$ such that the closed-loop system satisfies the constraints while meeting asymptotic stability criterion in $X_{T}$

Let $X_{N}$ be the set of feasible initial conditions such that there is an input $\mathbf{u}_{0 \cdot N}^{*}$ satisfying the constraints in (19) at time $t_{0}$. From the dual-mode principle [28, 29], $X_{N}$ is an invariant subsets of $X$ and satisfies that $X_{N} \supseteq X_{N-1} \supseteq \Lambda \supseteq X_{1} \supseteq X_{0}=X_{T}$.

Theorem 1: Under Assumption 1, the origin of the closed-loop system (20) is asymptotically stable with $X_{N}$ as a region of attraction.

Proof: We first prove recursive feasibility of (20) and then show asymptotic stability of (22).

Part 1: Consider the nominal system (1) and any $x_{0} \in X_{N} \backslash X_{T}$ at sampling time $t_{0}$. We compute the optimal control input $\mathbf{u}_{0 \cdot N}^{*}$ to (19) and apply it to the system until the time $t_{N}$ arrives. Due to the nominal system and satisfaction of the constraints in (19), it is derived that the system in the closed-loop with $u(t)=\mathbf{u}_{0: N}^{*}$, $t_{0} \leq t \leq t_{N}$, starting from $x_{0}$ enters $X_{T}$ within $N$-time periods, i.e. $x\left(t_{N}\right)=\left(t_{N} ; x_{0}, \mathbf{u}_{0: N}^{*}\right) \in X_{T}$.

Without loss of generality, pick any $x_{k} \in X_{T}$ at sampling time $t_{k}$. Let $\theta^{*}\left(t_{k}\right)$ be optimal solution to (20) at $t_{k}$, with associated input $\mathbf{u}_{k: k+N}^{*}$. Applying $\mathbf{u}_{k: k+N}^{*}$ to the system (1), we have $x\left(t_{k+1}\right)=\varphi\left(t_{k+1} ; x_{k}, \mathbf{u}_{k \cdot k+N}^{*}\right) \in X_{T}$ due to the invariance of $X_{T}$. From the construction of $X_{T}$, there exists a $\theta \in \Theta$ such that $u(t)=h(x(t), \theta) \in U$ and $\varphi\left(t ; x_{k+1}, \mathbf{u}_{k+1: k+1+N}^{*}\right) \subseteq X$ for all $t \in\left[t_{k+1}, t_{k+1+N}\right]$ and $\varphi\left(t_{k+N} ; x_{k+1}, \mathbf{u}_{k+1: k+1+N}^{*}\right) \in X_{T}$. Namely, $\theta$ is a feasible solution to (20) at next time $t_{k+1}$, which leads to recursive feasibility of (20).

Part 2: From Part 1, it is known that starting from any $x_{0} \in X_{N} \backslash X_{T}$, the system (22) applied by solving (19) enters $X_{T}$ within $N$-time periods, i.e. $x\left(t_{N}\right) \in X_{T}$. Then the MO-EMPC is switched to solve (20).

For any $x\left(t_{k+N}\right) \in X_{T}$ at sampling time $t_{k+N}$, we have $u^{*}(t)=h\left(x(t), \theta^{*}\left(t_{k}\right)\right)$ for $t \in\left[t_{k+N}, t_{k+N+1}\right]$ by solving (20). It is derived from (18) that the closed-loop system satisfies $V(x(t))<$ $V\left(x\left(t_{k+N}\right)\right)$ and hence $x(t) \in X_{T}$ for all $t \in\left[t_{k+N}, t_{k+N+1}\right]$. At the next time $t_{k+N+1}$, compute $u^{*}(t)=h\left(x(t), \theta^{*}(t)\right), t \in\left[t_{k+N+1}\right.$, $t_{k+N+2}$ ] to (20) with the state $x\left(t_{k+N+1}\right)$. Using again (18), we have $V(x(t))<V\left(x\left(t_{k+N+1}\right)\right)$ for all $t \in\left[t_{k+N+1}, t_{k+N+2}\right]$. By repeating the process, the system (22) satisfies $\lim _{t_{k} \rightarrow \infty} V(x(t))=0$ because of the positive definiteness of $V(x)$. Therefore, the closedloop states starting from $x\left(t_{k+N}\right) \in X_{T}$ satisfy $x\left(t_{k}\right) \rightarrow 0$ as $t_{k} \rightarrow$ $\infty$. Integrating the control input of (19), we conclude that the closed-loop system starting from any $x\left(t_{0}\right) \in X_{N}$ converges to the origin. Namely, asymptotic stability of the closed-loop system is established with $X_{N}$ as a region of attraction.

Remark 5: From the practical points of view, it is desired that the stability properties of systems in the closed-loop with controllers are not related to their criteria. Algorithm 1 (Fig. 2) satisfies such requirement to some extent. Specially, recursive feasibility and stability of (22) are independent of the choice of $N$ and $L(x, u)$. Hence, we can adjust online these terms to achieve better performances without causing stability concerns. In addition, from the proof of Theorem 1, the stability properties of (22) can be guaranteed by a feasible solution. Hence, the computational burden of solving both
(19) and (20) can be reduced at the expense of the optimality of the OCPs.

Theorem 2: Consider the closed-loop system (22) within $X_{N}$. Under Assumption 1, the closed-loop average performance of $L(x, u)$ satisfies that

$$
\lim _{k \rightarrow \infty} \sup \frac{1}{t_{k}} \int_{t_{0}}^{t_{k}} L\left(x(t), u^{m o}(t)\right) \mathrm{d} t=L\left(x_{s}^{c}, u_{s}^{c}\right) .
$$

Proof: Since the system (20) asymptotically converges to the equilibrium point $\left(x_{S}^{c}, u_{S}^{c}\right)$, for any $\varepsilon>0$ there exists a number $t_{\check{N}}=t_{0}+\check{N} \delta, \check{N} \in \mathbf{I}_{\geq 1}$ such that

$$
\left\|L\left(x(t), u^{m o}(t)\right)-L\left(x_{s}^{c}, u_{s}^{c}\right)\right\|<\varepsilon / 2, \quad \forall t \geq t_{\breve{N}} .
$$

For any $t_{k} \geq t_{\check{N}}$, then it is obtained that

$$
\begin{aligned}
& \left\|\int_{t_{0}}^{t_{k}} L\left(x(t), u^{m o}(t)\right) \mathrm{d} t-t_{k} L\left(x_{s}^{c}, u_{s}^{c}\right)\right\| \\
& \leq \int_{t_{0}}^{t_{\breve{N}}}\left\|L\left(x(t), u^{m o}(t)\right)-L\left(x_{s}^{c}, u_{s}^{c}\right)\right\| \mathrm{d} t \\
& +\int_{t_{\widehat{N}}}^{t_{k}}\left\|L\left(x(t), u^{m o}(t)\right)-L\left(x_{s}^{c}, u_{s}^{c}\right)\right\| \mathrm{d} t
\end{aligned}
$$

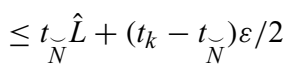

where $\hat{L}=\sup _{t \in\left[t_{0}, t_{N}\right]}\left\|L\left(x(t), u^{m o}(t)\right)-L\left(x_{s}^{c}, u_{s}^{c}\right)\right\|$ is finite. Let $t_{N *}=2 t_{\check{N}}(\hat{L}-\varepsilon / 2) / \varepsilon$. Then for any $t_{k}>t_{N *}$, we have

$$
\left\|\frac{1}{t_{k}} \int_{t_{0}}^{t_{k}} L\left(x(t), u^{m o}(t)\right) \mathrm{d} t-L\left(x_{s}^{c}, u_{s}^{c}\right)\right\| \leq(\hat{L}-\varepsilon / 2) t_{\breve{N}} / t_{k}+\varepsilon / 2<\varepsilon .
$$

From [12], this shows that (23) holds.

\section{Examples}

Two examples are used to illustrate effectiveness of the results presented here. For both examples, all optimisation problems had been solved by the function of fmincon in MATLAB 2014a on the computer of an Intel ${ }^{\circledR} \mathrm{Xeon}^{\circledR} \mathrm{CPU}$ with $3.2 \mathrm{GHz}$ and $8 \mathrm{~GB}$ RAM, and Euler's first-order approximation is employed for all derivatives.

\subsection{Example 1}

Consider an isothermal chemical reactor system [4]

$$
\begin{aligned}
\frac{\mathrm{d} c_{1}}{\mathrm{~d} t} & =\frac{Q_{i n}}{V_{R}}\left(c_{1 i n}-c_{1}\right)-\sigma c_{1}^{2} \\
\frac{\mathrm{d} c_{2}}{\mathrm{~d} t} & =\frac{Q_{i n}}{V_{R}}\left(c_{2 i n}-c_{2}\right)+\sigma c_{2}^{2}
\end{aligned}
$$

where $c_{1}$ and $c_{2}$ are the molar concentrations of species $A_{1}$ and $A_{2}$, respectively, $c_{1 \text { in }}$ and $c_{2 \text { in }}$ are the feed concentrations of $A_{1}$ and $A_{2}$, and $Q_{i n}$ is the flow through the reactor. The volume of the reactor $V_{R}=10 \mathrm{~L}$ and the rate constant $\sigma=1.2 \mathrm{l} /(\mathrm{mol} \cdot \mathrm{s})$. The concentrations $c_{1}$ and $c_{2}$ are the states and the flow $Q_{i n}$ is the control. The constraints are set to $x_{i} \in[0,1]$ for $i=1,2$ and $u \in[1,20]$. We consider two economic objectives of interest. The first objective is to maximise the amount of $A_{2}$ in the reactor, i.e. minimise economic stage cost $L_{1}(x, u)=-x_{2} u$. The second objective is to minimise the amount of $A_{1}$, i.e. minimise economic stage cost $L_{2}(x, u)=x_{1} u$. The stage cost vector is defined as $L(x, u):=\left[L_{1}(x, u), L_{2}(x, u)\right]^{\mathrm{T}}$. The control goal of the system is to minimise $L_{1}(x, u)$ and $L_{2}(x, u)$ 


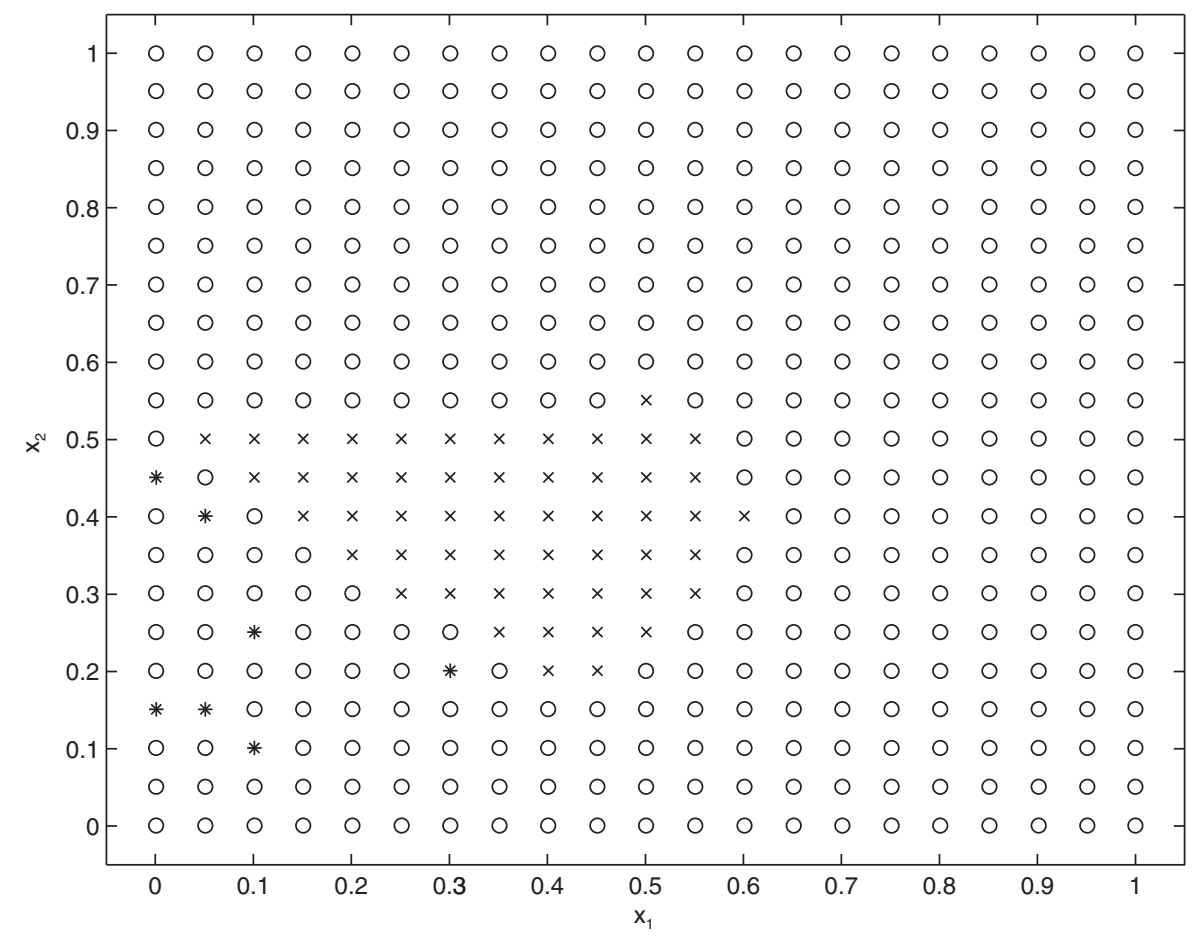

Fig. 3 Space distribution of four cases with respect to $A v\left\{L_{i}(x, u)\right\}$ applied by UMPC and SMPC, where '*' denotes Case 1, 'o' Case 2, ' $\times$ ' Case 3 and '+' Case 4

simultaneously, while stabilising the system to some economically optimal steady-state point in the face of the constraints.

According to the steady-state optimisation problem (4), the steady-state utopia point of $L(x, u)$ is computed as $L_{s}^{*}=$ $[-5.9349,0.25]^{\mathrm{T}}$ associated with the economically optimal steadystate solutions $\left(x_{s}^{1 *}, u_{s}^{1 *}\right)=(0.7033,0.2967,20.0)$ and $\left(x_{s}^{2 *}, u_{s}^{2 *}\right)=$ $(0.2500,0.7500,1.0)$, respectively. Then the compromised solution is determined as $\left(x_{s}^{c}, u_{s}^{c}\right)=(0.4645,0.5355,4.8358)$. By evaluating (8) offline, the ultimate utopia point of $L(x, u)$ is computed as $L_{u}^{*}=[-9.9288,0.1567]^{\mathrm{T}}$. Clearly, we have $L_{u, i}^{*}<L_{s, i}^{*}$ for $i=1$ and 2, which is due to the fact that the computation of $L_{u}^{*}$ takes into account the transient response of (27).

Let the system $(A, B)$ be the linearised model of (27) at the point $\left(x_{s}^{c}, u_{s}^{c}\right)$. Solving the Riccati equation

$$
A^{\mathrm{T}} P+P A-2 \theta_{0} P B B^{\mathrm{T}} P=-q I
$$

with $\theta_{0}=0.1$ and $q=10$, we have a positive definite matrix solution

$$
P=\left[\begin{array}{cc}
6.9854 & 5.5310 \\
5.5310 & 10.3259
\end{array}\right]
$$

Then we construct a locally Lipschitz control law

$$
u=-\theta K\left(x-x_{s}^{c}\right)+u_{s}^{c}, \quad \theta \geq \theta_{0}
$$

where $K=B^{\mathrm{T}} P=[0.0348,-0.1148]$. Let $V(z)=z^{\mathrm{T}} P z$ with the shifted variable $z=x-x_{s}^{c}$. We obtain the level set $X_{T}\left(x_{s}^{c}\right)=$ $\left\{z \in R^{2} \mid V(z) \leq 1.5072\right\}$ of $V(z)$, which is an invariant set of the system (27) in the closed-loop with (14). For computation consideration, the upper bound of $\theta$ is set to 1000 .

Denote by SMPC and UMPC the steady-state and the ultimate utopia-tracking MO-MPC controllers, respectively. The sampling period are chosen as $\delta=0.1 \mathrm{~s}$ and the prediction horizon $t_{N}=5 \delta$. Let $A v\left\{L_{i}(x, u)\right\}$ be the average performance of $L_{i}(x, u), i=1$, 2 , evaluated over the simulation horizon. To compare the differences of $A v\left\{L_{i}(x, u)\right\}$ applied separately by UMPC and SMPC, we consider the following four cases:
Table 1 Average performances of $L_{1}(x, u)$ and $L_{2}(x, u)$ applied by SMPC and UMPC from eight initial states

\begin{tabular}{lccllll}
\hline & \multicolumn{2}{c}{$A v\left\{L_{1}(x, u)\right\}$} & & \multicolumn{2}{c}{$A v\left\{L_{2}(x, u)\right\}$} & \\
\cline { 2 - 3 } Initial states & SMPC & UMPC & & SMPC & UMPC & Case \\
\hline $\mathrm{a}(0.50,0.90)$ & -3.1731 & -3.4875 & & 2.3313 & 2.9327 & 2 \\
$\mathrm{~b}(0.90,0.70)$ & -3.3943 & -3.6728 & & 2.7750 & 3.2163 & 2 \\
$\mathrm{c}(0.65,0.50)$ & -2.7872 & -2.8731 & & 2.4160 & 2.5923 & 2 \\
$\mathrm{~d}(0.50,0.30)$ & -2.2972 & -2.2972 & & 2.2610 & 2.2610 & 3 \\
$\mathrm{e}(0.25,0.70)$ & -2.6440 & -2.8117 & & 2.1703 & 2.3307 & 2 \\
$\mathrm{f}(0.14,0.24)$ & -2.0221 & -2.0332 & & 2.2204 & 2.2007 & 1 \\
$\mathrm{~g}(0.10,0.10)$ & -1.9729 & -1.9737 & & 2.4121 & 2.4055 & 1 \\
$\mathrm{~h}(0.25,0.30)$ & -2.0679 & -2.0679 & & 2.1438 & 2.1438 & 3 \\
\hline
\end{tabular}

Case 1: UMPC outperforms SMPC in terms of both $A v\left\{L_{1}(x, u)\right\}$ and $A v\left\{L_{2}(x, u)\right\}$.

Case 2: UMPC outperforms SMPC in at least one of $A v\left\{L_{1}(x, u)\right\}$ and $A v\left\{L_{2}(x, u)\right\}$.

Case 3: UMPC and SMPC have equal performances.

Case 4: SMPC outperforms UMPC in terms of both $A v\left\{L_{1}(x, u)\right\}$ and $A v\left\{L_{2}(x, u)\right\}$.

Fig. 3 presents the distribution of these cases in the operation space of system (27). It is depicted by gridding the state constraint set $[0,1] \times[0,1]$ in increments of 0.05 , which leads to a total of 141 initial points. As we can see, there is no point in Fig. 3 for Case 4; but there are seven points for Case 1. In particular, Table 1 tabulates the values of $A v\left\{L_{i}(x, u)\right\}$ starting from eight initial states (a)-(h), obtained by separately applying SMPC and UMPC. It is observed that for the initial states (a), (b), (c) and (e), the average performances of $L_{1}(x, u)$ by UMPC are better than those by SMPC; however, the average performances of $L_{2}(x, u)$ by UMPC are worse than those by SMPC. Nevertheless, we can see that for the initial states (d) and (h), the average performances of both $L_{1}(x, u)$ and 
$L_{2}(x, u)$ by UMPC are equal to those by SMPC; and for the initial states (f) and (g), the average performances of both $L_{1}(x, u)$ and $L_{2}(x, u)$ by UMPC are better than those by SMPC. These observations suggest that the closed-loop average performances of stage costs obtained by applying UMPC are no worse than those by SMPC.

Fig. 4 shows the closed-loop state phase profiles applied by UMPC starting from the initial states (a)-(h), where the ' $*$ ' is the economically optimal equilibrium state $x_{s}^{c}$. Moreover, Fig. 5 depicts the state responses and control inputs of (27) in the closedloop with UMPC with initial state (a) (the solid lines) and (b) (the dashed lines). From the graphs in Figs. 4 and 5, it is clear that from the different initial states, the state trajectories of (27) in the closed-loop with UMPC are asymptotically convergent to the equilibrium state while satisfying the constraints for all times. Note that it is not easy to ensure that the system (27) is dissipative with

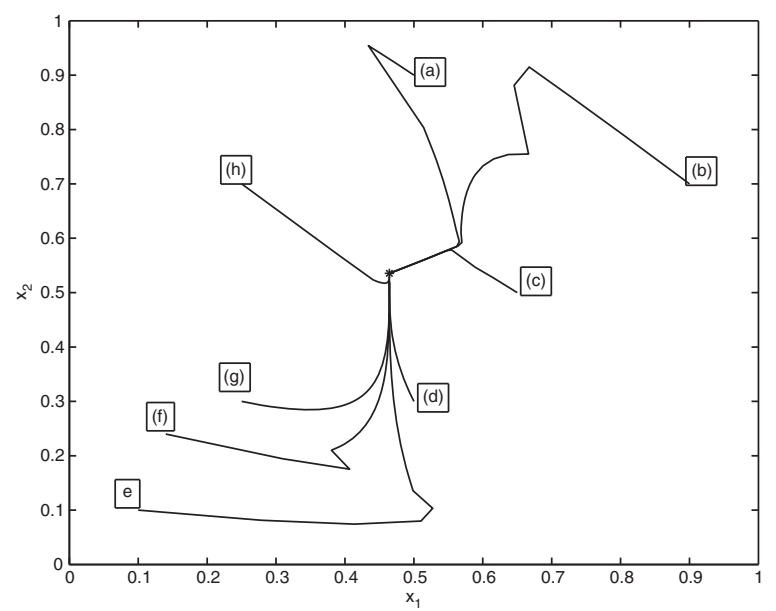

Fig. 4 Phase profiles of the closed-loop system under UMPC from different initial concentrations
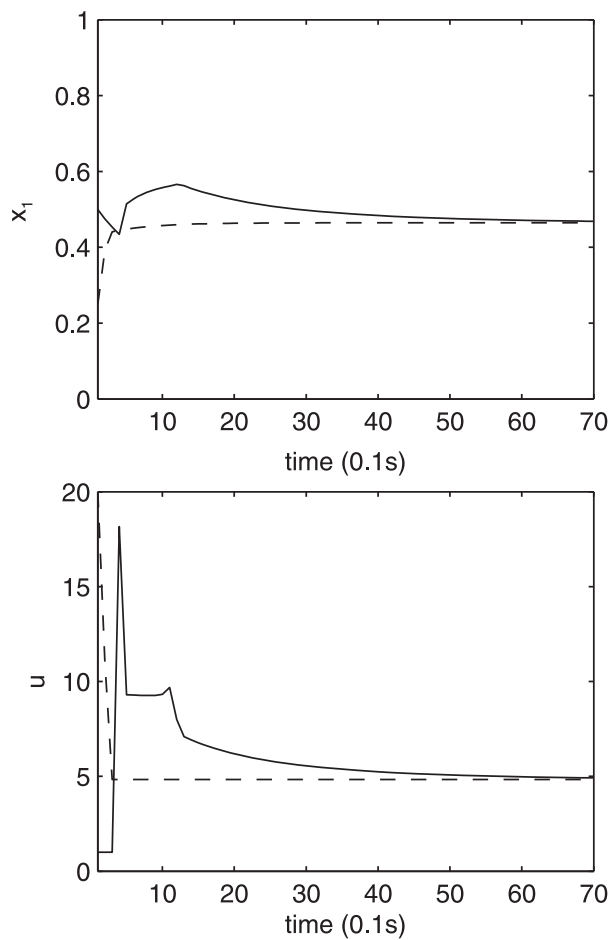

respect to $L_{1}(x, u)$ and $L_{2}(x, u)$. On the other hand, it is seen from Fig. 5 that the computed control input may be not smooth. This limits the performance of the UMPC controller from the viewpoint of smoothness of signals.

To show the differences resulted from using the terminal region and terminal state constraints, Table 2 tabulates the shortest prediction horizon $t_{N \text { min }}$ that is used to guarantee the feasibility of the OCPs at initial time $t_{0}=0$. It is seen that $t_{N \text { min }}$ associated with the terminal region constraint is smaller than that with the terminal state constraint. This difference is consistent with the well-accepted conclusion of traditional single objective NMPC and also implies that the set of initial feasible states corresponding to the terminal region constraint is bigger than that to the terminal state constraint if the prediction horizons are selected to be equal.

\subsection{Example 2}

In order to further demonstrate the proposed MO-EMPC strategy, we consider a more realistic irreversible exothermic reaction system $[25,28]$

$$
\begin{aligned}
& \dot{C}_{1}=\frac{q}{V}\left(C_{f}-C_{1}\right)-k_{0} C_{1} \exp \left(-\frac{E / R}{T_{r}}\right) \\
& \dot{T}_{r}=\frac{q}{V}\left(T_{f}-T_{r}\right)-\frac{\Delta H}{\rho C_{p}} k_{0} C_{1} \exp \left(-\frac{E / R}{T_{r}}\right)+\frac{U_{A}}{V \rho C_{p}}\left(T_{c}-T_{r}\right)
\end{aligned}
$$

where $C_{1}$ is the concentration of production $A_{1}$ in the reactor, $T_{r}$ the reactor temperature and $T_{c}$ the temperature

Table 2 Shortest prediction horizon for ensuring feasibility of the OCPs at $t_{0}$

\begin{tabular}{ccccccccc}
\hline & $\mathrm{a}$ & $\mathrm{b}$ & $\mathrm{c}$ & $\mathrm{d}$ & $\mathrm{e}$ & $\mathrm{f}$ & $\mathrm{g}$ & $\mathrm{h}$ \\
\hline UMPC & $\delta$ & $2 \delta$ & $\delta$ & $\delta$ & $\delta$ & $\delta$ & $\delta$ & $\delta$ \\
SMPC & $54 \delta$ & $56 \delta$ & $48 \delta$ & $26 \delta$ & $57 \delta$ & $43 \delta$ & $43 \delta$ & $28 \delta$ \\
\hline
\end{tabular}
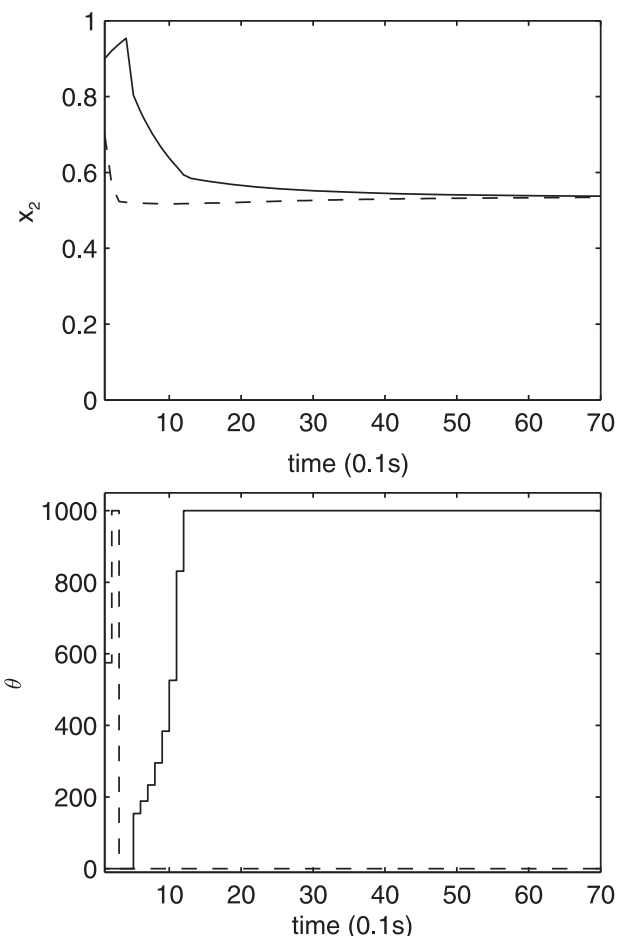

Fig. 5 State responses and control inputs of the closed-loop system under UMPC and the profiles of $\theta$, associated with initial state $a$ (Solid lines) $b$ (Dashed lines) 

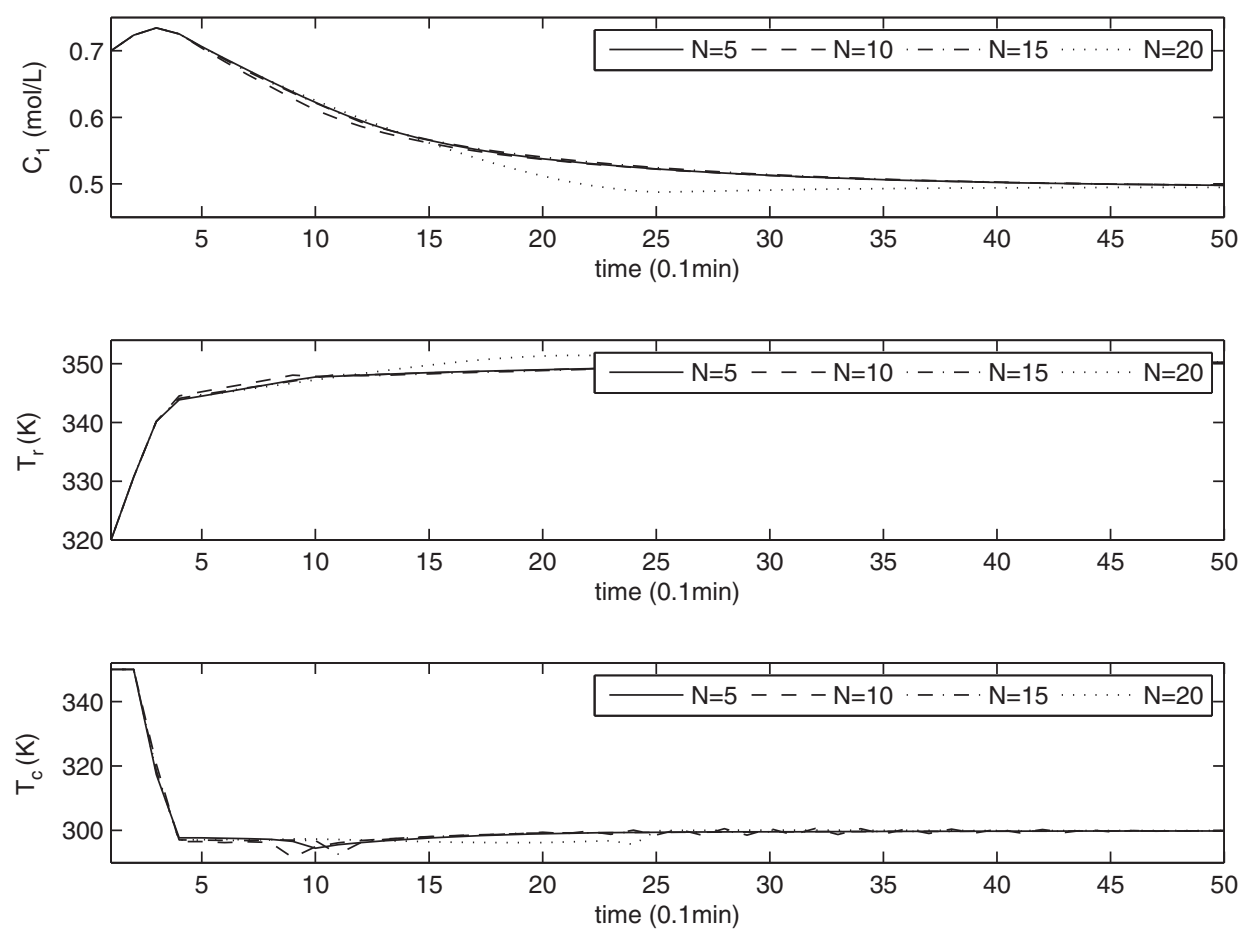

Fig. 6 State responses and control inputs of the closed-loop system

of coolant stream. The model parameters are chosen as follows: $q=100 \mathrm{~L} / \mathrm{min}, T_{f}=350 \mathrm{~K}, C_{f}=1 \mathrm{~mol} / 1, V=100 \mathrm{~L}$, $\rho=1000 \mathrm{~g} / \mathrm{L}, C_{p}=0.239 \mathrm{~J} / \mathrm{g} \cdot \mathrm{K}, \Delta H=-5 \times 10^{4} \mathrm{~J} / \mathrm{mol}, E / R=$ $8750 \mathrm{~K}, k_{0}=7.2 \times 10^{10} \mathrm{~min}^{-1}$ and $U_{A}=5 \times 10^{4} \mathrm{~J} / \mathrm{min} \cdot \mathrm{K}$. Let the state vector be $x=\left[x_{1}, x_{2}\right]^{\mathrm{T}}=\left[C_{1}, T_{r}\right]^{\mathrm{T}}$ and the control variable $u=T_{c}$. System (30) is subject to the following constraints

$$
X=[0,1] \times[300,370], \quad U=[280,350] .
$$

This system is required to be operated at the maximal production rate of $A_{1}$

$$
r(x)=k_{0} C_{1} \exp \left(-\frac{E / R}{T_{r}}\right)=k_{0} x_{1} \exp \left(-\frac{E / R}{x_{2}}\right)
$$

and the minimal energy usage/removal, $T_{c}-\left(T_{r}-T_{f}\right)^{2}$. Let $L_{1}(x, u)=-r(x)$ and $L_{2}(x, u)=u-\left(x_{2}-T_{f}\right)^{2}$. Then the goal is to minimise $L_{1}(x, u)$ and $L_{2}(x, u)$ simultaneously, while stabilising the system to some economically optimal steady-state point and satisfying (31).

Let $L(x, u)=\left[L_{1}(x, u), L_{2}(x, u)\right]^{\mathrm{T}}$. By solving (4), the steadystate utopia point of $L(x, u)$ is computed as $L_{s}^{*}=[-0.7943$, $299.9547]^{\mathrm{T}}$ with the optimal solutions $\left(x_{s}^{1 *}, u_{s}^{1 *}\right)=(0.2057,370.0$, $300.1261)$ and $\left(x_{s}^{2 *}, u_{s}^{2 *}\right)=(0.4973,350.1530,299.9547)$, respectively. Then the compromised solution $\left(x_{s}^{c}, u_{s}^{c}\right)=(0.4948,350.290$, 299.9129). By evaluating (8) offline, the ultimate utopia point is computed as $L_{u}^{*}=[-0.7945,299.980]^{\mathrm{T}}$ and satisfies that $L_{u, i}^{*}<$ $L_{s, i}^{*}$ for $i=1$ and 2 . Using the same method as Example 1, we have a locally Lipschitz control law (14) with $\theta_{0}=0.5$ and $K=[114.0403 .3826]$, and the set $X_{T}\left(x_{s}^{c}\right)=\left\{z \in R^{2} \mid V(z) \leq 210\right\}$ with $V(z)=z^{T} P z$ and

$$
P=\left[\begin{array}{cc}
2543.2857 & 54.5133 \\
54.5133 & 1.6169
\end{array}\right]
$$

The upper bound of $\theta$ in (29) is set to 1000 .

Let the sampling period $\delta=0.1 \mathrm{~min}$ and pick an initial state $(0.7 \mathrm{~mol} / \mathrm{l}, 320 \mathrm{~K})$. Fig. 6 depicts the state responses and control
Table 3 Average economic performances and computational times for different values of $t_{N}$

\begin{tabular}{lcccc}
\hline$t_{N}$ & $5 \delta$ & $10 \delta$ & $15 \delta$ & $20 \delta$ \\
\hline$A v\left\{L_{1}(x, u)\right\}$ & -0.4844 & -0.4861 & -0.4838 & -0.4961 \\
$A v\left\{L_{2}(x, u)\right\}$ & 331.9102 & 331.1098 & 331.8694 & 331.7117 \\
$T_{\max }(\mathrm{ms})$ & 883.1 & 966.4 & 1175.8 & 1363.0 \\
$T_{\text {ave }}(\mathrm{ms})$ & 35.6 & 40.3 & 42.8 & 48.1 \\
\hline
\end{tabular}

inputs of (30) in the closed-loop with the proposed MO-EMPC with the prediction horizon $t_{N}=5 \delta$ (solid lines), $t_{N}=10 \delta$ (dashed lines), $t_{N}=15 \delta$ (dash-dotted lines), and $t_{N}=20 \delta$ (dotted lines). As expected that for different selection of the prediction horizon, the closed-loop trajectories of production concentration $C_{1}$, reactor temperature $T_{r}$ and coolant stream temperature $T_{c}$ obtained by applying the proposed MO-EMPC are asymptotically convergent to the compromised equilibrium point $\left(x_{s}^{c}, u_{s}^{c}\right)$ in the presence of the state and control constraints (31). However, these closedloop trajectories approach the equilibrium point in different ways. In particular, the selection of a larger prediction horizon speeds up the response of the closed-loop system (e.g. see the dotted lines).

In what follows, we quantitatively assess the average economic performances over a simulation horizon $t_{\text {sim }}=50 \delta$ and the computational times with respect to the prediction horizon $t_{N}$. Table 3 tabulates average performances and computational times by applying the proposed MO-EMPC for different prediction horizons. It can be seen that the obtained average performances $A v\left\{L_{1}(x, u)\right\}$ and $A v\left\{L_{2}(x, u)\right\}$ are not necessarily improved by extending or reducing the prediction horizon $t_{N}$. In other words, the average performance obtained by applying the proposed MO-EMPC is not a monotone function on $t_{N}$. However, the maximal computational time $T_{\max }$ and the average computational time $T_{\text {ave }}$, over the simulation horizon, are reduced when we select a shorter length of $t_{N}$. Hence, from Fig. 6 and Table 3 the prediction horizon of the proposed MO-EMPC should be determined by the trade-off among closed-loop responses, average performances and computational times. 


\section{Conclusion}

In this work, an ultimate utopia-tracking MO-EMPC scheme was presented to tackle multiobjective economic optimisation problems of constrained non-linear systems. The concept of ultimate utopia point was introduced to address conflicting economic criteria. Then the utopia-tracking optimisation problem was formulated into a dual-mode control problem, where the MO-EMPC law is determined by minimising the distance of its cost function vector to the ultimate utopia point. The asymptotic stability and average performance of the closed-loop system were established using a terminal region constraint and the assumption of controllability. The simulation results demonstrated the good properties of the proposed MO-EMPC to deal with conflicting economic criteria.

\section{Acknowledgments}

This work was supported by the National Natural Science Foundation of China under grant no. 61374111 and Public Welfare Technology Application Research Project of Zhejiang Province under grant no. $2015 \mathrm{C} 31057$.

\section{References}

1 Ellis, M., Durand, H., Christofides, P.D.: 'A tutorial review of economic model predictive control methods', J. Process Control, 2014, 24, (8), pp. $1156-1178$

2 Mayne, D.Q.: 'Model predictive control: recent developments and future promise', Automatica, 2014, 50, pp. 2967-2986

3 Amrit, R., Rawlings, J.B., Angeli, D.: 'Economic optimization using model predictive control with a terminal cost', Annu. Rev. Control, 2011, 35, (2), p. 178186

4 Diehl, M., Amrit, R., Rawlings, J.B.: 'A Lyapunov function for economic optimizing model predictive control', IEEE Trans. Autom. Control, 2011, 56, (3), pp. $703-707$

5 Heidarinejad, M., Liu, J., Christofides, P.D.: 'Economic model predictive control of nonlinear process systems using Lyapunov techniques', AIChE J., 2012, $\mathbf{5 8}$, pp. $855-870$

6 Huang, R., Harinath, E., Biegler, L.T.: 'Lyapunov stability of economically oriented NMPC for cyclic processes', J. Process Control, 2011, 21, (4), pp. 501-509

7 Angeli, D., Amrit, R., Rawlings, J.B.: 'On average performance and stability of economic model predictive control', IEEE Trans. Autom. Control, 2012, 57, (7), pp. $1615-1626$
8 Fagiano, L., Teel, A.R.: 'Generalized terminal state constraint for model predictive control', Automatica, 2013, 49, (9), pp. 2622-2631

9 Guay, M., Adetola, V.: 'Adaptive economic optimising model predictive control of uncertain nonlinear systems', Int. J. Control, 2013, 86, (8), pp. 1425-1437

10 Grúne, L.: 'Economic receding horizon control without terminal constraints', Automatica, 2013, 49, (3), pp. 725-734

11 Múller, M.A., Angeli, D., Allgówer, F. et al.: 'Convergence in economic model predictive control with average constraints', Automatica, 2014, 50, (12), pp. $3100-3111$

12 Ellis, M., Christofides, P.D.: 'On finite-time and infinite-time cost improvement of economic model predictive control for nonlinear systems', Automatica, 2014, 50, (10), pp. 2561-2569

13 Limon, D., De la Pereira, M., Pena, D.M. et al. 'Single-layer economic model predictive control for periodic operation', J. Process Control, 2014, 24, (8), pp. $1207-1224$

14 Rawlings, J.B., Angeli, D., Bates, C.N.: 'Fundamentals of economic model predictive control', Proc. 51st IEEE Conf. Decision and Control, Maui, Hawaii, 2012, 0, pp. 3851-3861

15 Maree, J.P., Imsland, L.: 'On multi-objective economic predictive control for cyclic process operation', J. Process Control, 2014, 24, (8), pp. 1328-1336

16 Ehrgott, M.: 'Multicriteria optimization' (Springer, Berlin Heidelberg, 2005, 2nd edn.)

17 Vito, D., Scattolini, R.: 'A receding horizon approach to the multiobjective control problem', Proc. 46th IEEE Conf. Decision and Control, New Orleans, $L A, 2007$, 0, pp. 6029-6034

18 Bemporad, A., de la Pena Munoz, D.: 'Multiobjective model predictive control', Automatica, 2009, 45, pp. 2823-2830

19 Vallerio, M., Van Impe, J., Logist, F.: 'Tuning of NMPC controllers via multi-objective optimisation', Comput. Chem. Eng., 2014, 61, pp. 38-50

20 Flores-Tlacuahuac, A., Morales, P., Rivera-Toledo, M.: 'Multiobjective Nonlinear model predictive control of a class of chemical reactor', Ind. Eng. Chem. Res., 2012, 51, (17), pp. 5891-5899

21 Zavala, V.M., Flores-Tlacuahuac, A.: 'Stability of multiobjective predictive control: an utopia-tracking approach', Automatica, 2012, 48, (10), pp. 2627-2632

22 He, D., Wang, L., Yu, L.: 'Multi-objective nonlinear predictive control of process systems: a dual-mode tracking control approach', J. Process Control, 2015, 25, pp. $142-151$

23 Khalil, H.K.: 'Nonlinear systems' (Prentice Hall, Inc., 2002)

24 Maree, J.P., Imsland, L.: 'Multi-objective predictive control for non steady-state operation', Proc. 2013 European Control Conf., Zurich, Switzerland, 2013, 0, pp. 1541-1546

25 El-Farra, N.H., Christofides, P.D.: 'Bounded robust control of constrained multivariable nonlinear processes', Chem. Eng. Sci., 2003, 58, pp. 3025-3047

26 He, D., Yu, L., Song, X.: 'Optimized-based stabilization of constrained nonlinear systems: a receding horizon approach', Asian J. Control, 2014, 16, (6), pp. 1693-1701

27 Sepulchre, R., Jankovic, M., Kokotovic, P.V.: 'Constructive nonlinear control' (Springer, London, 1997)

28 Limon, D., Bravo, J.M., Alamo, T. et al.: 'Robust MPC of constrained nonlinear systems based on interval arithmetic', IEE Proc. Control Theory Appl., 2005 , 152, (3), pp. 325-332

29 Michalska, H., Mayne, D.Q.: 'Robust receding horizon control of constrained nonlinear systems', IEEE Trans. Autom. Control, 1993, 38, (11), pp. 1623-1633 